\section{A multicentre, randomised controlled study of enteric-coated mycophenolate sodium for the treatment of relapsed or resistant proliferative lupus nephritis: an Asian experience}

Sirirat Anutrakulchai, ${ }^{1}$ Thanachai Panaput, ${ }^{2}$ Jeerapat Wongchinsri, ${ }^{3}$ Somchai Chaishayanon, ${ }^{3}$ Bancha Satirapoj, ${ }^{4}$ Opas Traitanon, ${ }^{5}$ Warabhorn Pima, ${ }^{6}$ Chutima Rukrung, ${ }^{7}$ Bandit Thinkhamrop, ${ }^{8}$ Yingyos Avihingsanon, ${ }^{9,10}$ for the CONTROL-Lupus study group

To cite: Anutrakulchai S, Panaput T, Wongchinsri J, et al. A multicentre, randomised controlled study of enteric-coated mycophenolate sodium for the treatment of relapsed or resistant proliferative lupus nephritis: an Asian experience. Lupus Science \& Medicine 2016;3:e000120. doi:10.1136/lupus-2015000120

- Additional material is available. To view please visit the journal (http://dx.doi.org/ 10.1136/lupus-2015000120).

Received 31 July 2015 Revised 26 November 2015 Accepted 13 December 2015

\section{CrossMark}

For numbered affiliations see end of article.

\section{Correspondence to} Professor Yingyos Avihingsanon; Yingyos.a@gmail.com

\section{ABSTRACT}

Objective: The optimal treatment of relapse or resistant lupus nephritis (LN) is still unclear. Mycophenolate might be an alternative therapy to avoid toxicities of cyclophosphamide (CYC). This study was aimed to compare enteric-coated mycophenolate sodium (ECMPS) versus intravenous CYC as an induction therapy. Methods: The study was a 12-month period of multicentre, open-labelled randomised controlled trial. Fifty-nine patients who had relapsed $(36 \%)$ or who were resistant to previous CYC treatment $(64 \%)$ and all who were biopsy-proven class III/IV, were randomised into CYC $(n=32)$ and EC-MPS groups $(n=27)$. The CYC group received intravenous CYC $0.5-1 \mathrm{~g} / \mathrm{m}^{2}$ monthly and the EC-MPS group was treated with EC-MPS $1440 \mathrm{mg} /$ day for first 6 months. After induction therapy, both groups received EC-MPS $720 \mathrm{mg} /$ day until the end of study at 12 months.

Results: The study was prematurely terminated due to high rate of serious adverse events in CYC arm. Death and serious infections were observed more in the CYC group ( $15.6 \%$ in CYC and $3.5 \%$ in EC-MPS; $p=0.04$ ). The early discontinuation rates, mainly from serious infections, were significantly higher in CYC group (percentage differences of $16.9 ; 95 \% \mathrm{Cl} 1.3$ to 32.4 ). At the 12th month, both arms were comparable in terms of complete and partial remission rates $68 \%$ CYC and $71 \%$ EC-MPS) and times to remission (96 days CYC and 97 days EC-MPS). Composites of unfavourable outcomes (death, doubling of serum creatinine, non-remission and intolerance to treatment) were $46.9 \%$ and $37 \%$ in CYC and EC-MPS (risk difference $=9.84 ; p=0.44$ ).

Conclusions: EC-MPS may have comparable efficacy, but was better tolerated than CYC. EC-MPS should be an alternative choice of treatment for difficult-to-treat LN, particularly in CYC-experienced LN patients. Due to an early termination of the study, further clinical implementation could be cautiously used.

Trial registration number: Clinicaltrials.gov ID\#NCT01015456.

\section{KEY MESSAGES}

For difficult-to-treat LN, there is minimal evidences for the optimal treatment.

- Treatment with intravenous cyclophosphamide and steroids could increase risks of infectionrelated mortality in this setting.

- Enteric-coated mycophenolate sodium could be an alternative treatment of choice for this condition in Asian patients.

\section{INTRODUCTION}

Lupus nephritis (LN) is the leading cause of chronic glomerulonephritis and could rapidly develop into an end-stage renal disease. ${ }^{1-3}$ It is a common and severe form in patients with systemic lupus erythematosus among those of Asian ethnicity. ${ }^{4-7}$ The proliferative LN (the International Society of Nephrology and the Renal Pathology Society (ISN/RPS) class III/ IV) is common and has the worst prognosis. ${ }^{2} 8-11$ Relapse and resistance to therapy are the grave prognostic factors. ${ }^{3}{ }^{12-15}$ Although cyclophosphamide (CYC) is an effective treatment of choice, cumulative toxicities are its main limitation in repeated administration. In real-life practice, many patients were previously treated with CYC, and they have a relapse or have received certain dosages of CYC without improvement. In these so-called difficult-to-treat cases, patients were similarly exposed to steroids, CYC and other immunosuppressants.

Two forms of mycophenolic acid drugs including mycophenolate mofetil (MMF) and enteric-coated mycophenolate sodium (EC-MPS) are available. MMF was proven effective for the treatment of active $\mathrm{LN}$ in both 
induction and maintenance periods. ${ }^{16-21}$ Mycophenolate-based regimes provided equal remission rates to that of CYC, ${ }^{16} 1719$ 22-25 whereas mycophenolate therapy had lower flare rates than intravenous CYC. ${ }^{24} 26$ Furthermore, mycophenolate has resulted in fewer adverse events. For instance, ovarian failure, alopecia and leucopenia are more common in CYC users. ${ }^{16}{ }^{17}$ Nevertheless, mycophenolate remains not widely available in resource-limited settings. ${ }^{23} 27$

To date, only two case series have reported the efficacy of MMF in the treatment of relapsed LN or that of those resistant to treatment ${ }^{28} 29$ while the current authors reported, in a pilot study, successful EC-MPS therapy in resistant and patients with relapsed $\mathrm{LN}^{30}$ Six months of EC-MPS therapy provided an improvement in both clinical measurements and renal histopathology. Nevertheless, current evidence for the use of EC-MPS for the treatment of relapsed/resistant LN is lacking. In the guidelines, repeated treatment with either mycophenolate or CYC is suggested for relapse, but there is no consensus in treatment for those refractory to CYC. Possible strategies are first to switch to a mycophenolatebased regime ${ }^{31-33}$ or second to remain on an extended CYC course. ${ }^{12}{ }^{34}$ Nevertheless, no evidence in a comparable trial is available. This study was aimed to compare efficacy and safety between EC-MPS and CYC therapy for difficult-to-treat cases of $\mathrm{LN}$ in an investigatorinitiated, multicentre, randomised controlled trial in Thailand.

\section{METHODS}

Study design

From March 2010 through November 2012, six Thai medical centres participated in the study in accordance with the ethical principles of the Declaration of Helsinki, the Good Clinical Practice guidelines. The local medical ethics committees or institutional review boards approved the protocol and all patients provided written informed consents. This study was registered in the Clinical Trials registry (Clinicaltrials.gov ID\#NCT01015456).

A 12-month period of multicentre, open-labelled randomised controlled study comparing EC-MPS (Myfortic, Novartis, Basel, Switzerland) with intravenous CYC for the first 6 months of initial treatment in relapsed or the resistant type of proliferative LN was conducted. Then, all patients from both treatment arms received similar dosages of EC-MPS for maintenance treatment. The block of four randomisations for the treatment drugs was computerised and stratified by the type of clinical presentation (relapsed or resistant) and by centres. The data management and trial monitoring were conducted by the Medical University Research Network (MedResNet). The investigators have confidentiality agreements with the manufacturer (Novartis, Basel, Switzerland) as an investigator-initiated trial (CERL080ATH04T). The investigators had independent roles in the design of the trial or in the collection, analysis or interpretation of the data or the writing of the manuscript. The study was monitored for safety and efficacy of treatment by the Data Safety Monitoring Board (DSMB) and the committee designated stopping rules that included the rate of serious adverse events over $10 \%$ of any treatment group.

\section{Study participants}

Adults with an active LN, defined as a biopsy-proven proliferative LN according to ISN/RPS class III or IV with active lesions, were eligible to enrol in the trial if they were relapsed or resistant to prior CYC treatment. The relapsed and resistant cases were defined by criteria that included if the patients with active $\mathrm{LN}$ had either clinical features of an increase in serum creatinine $\geq 0.3 \mathrm{mg} / \mathrm{dL}$ or an increase in proteinuria $>1.5 \mathrm{~g} /$ day after previous renal remission of at least 6 months (relapsed cases) or after receiving three consecutive intravenous CYC treatments (resistant cases). In fact, both conditions had similar patient characteristics (see online supplementary tables S1-S3). The major exclusion criteria were sustained renal impairment (estimated glomerular filtration rate $\left.(\mathrm{eGFR})<25 \mathrm{~mL} / \mathrm{min} / 1.73 \mathrm{~m}^{2}\right)$ of longer than 3 months, severe extrarenal organ involvement, uncontrolled infection, cytopenia and pregnancy. The criteria for discontinuation from the study were severe infection, unable to tolerate studied drugs, declined renal function of over $50 \%$ or refusal to participate in the study.

\section{Study protocol and immunosuppressive treatment}

The study protocol consisted of a 2-week screening period and a biopsy-proven LN within 16 weeks prior to randomisation and a treatment period of 12 months for both parallel arms. Concerning infectious risk in those who received three consecutive doses prior to randomisation, CYC was withheld for at least 6 weeks before randomisation. All patients were on oral prednisolone $(0.7 \mathrm{mg} / \mathrm{kg} /$ day $)$ and were screened for active infection. The first group (arm A) received the induction-phase standard-dose intravenous CYC, so-called 'National Institute of Health regimen' $\left(0.5-1 \mathrm{~g} / \mathrm{m}^{2}\right.$ monthly for 6 months). ${ }^{35}$ The second group (arm B) was treated for the first 6 months with EC-MPS $1440 \mathrm{mg} /$ day. This dosage was equal to MMF (CellCept) $2 \mathrm{~g}$ /day and it was decided based on the Asian pharmacokinetics and randomised trials. ${ }^{23} \quad 30 \quad 36$ After 6 months of induction therapy, both groups received EC-MPS $720 \mathrm{mg}$ /day until the end of study at 12 months. In the case of crescentic formation of less than $30 \%$ of total glomeruli, methylprednisolone was given at $0.5-1 \mathrm{~g} /$ day for 3 days. Oral prednisolone was given at $0.7 \mathrm{mg} / \mathrm{kg} /$ day and decreased $5 \mathrm{mg}$ every 2-4 weeks, aiming to reach $20 \mathrm{mg}$ at the third month and $5 \mathrm{mg}$ at the sixth month.

Data collection that was composed of the baseline characteristics and monthly collection of blood pressure, complete blood counts, blood urea nitrogen, serum creatinine, serum albumin, urinalysis, $24 \mathrm{~h}$ urine protein, urine protein/creatinine ratios and eGFRs was measured by the chronic kidney disease epidemiology formula. 
Urine protein and serum creatinine analyses were performed at the central laboratory of the Chula Clinical Research Laboratory, Faculty of Medicine, Chulalongkorn University.

Remission was defined as stabilisation or improvement of renal function, resolution of urine sediment or cellular casts with improvement in terms of proteinuria, which could be categorised to (1) complete remission if proteinuria was $<0.5 \mathrm{~g} /$ day and (2) partial remission if proteinuria was $<1.5 \mathrm{~g} /$ day or a $>50 \%$ decrease from baseline and must have been $<3 \mathrm{~g} /$ day if baseline was $>3.5 \mathrm{~g} /$ day.

\section{Primary and secondary outcomes}

The primary outcomes of the study were the remission rates, times to achieve remission and a composition of unfavourable outcomes including intolerance to treatment, non-remission, doubling of serum creatinine, and death. The secondary outcomes were the percentages of nephrotic syndrome, renal impairment and adverse events related to the treatment protocol.

\section{Statistical analysis}

Initially the sample size was calculated based on a preliminary report that an overall response rate was $58 \%$ in the EC-MPS group and $35 \%$ in the CYC group. ${ }^{30}$ With a $10 \%$ drop-out rate, 140 patients (70 per treatment arm) were required to detect a difference of at least $20 \%$ with
$80 \%$ power between both treatment arms at a two-sided $\alpha$ level of 0.05 .

Since the rate of serious adverse events was $15.6 \%$ $(>10 \%)$ in CYC arm, the DSMB decided to terminate the study as written in the stopping rules. As a result, 59 participants (42\% of target number) were enrolled and analysed.

Baseline characteristics of both study groups are presented as mean \pm SD for continuous variables or medians with range for highly skewed data. For categorical variables, frequency count and percentages are presented. The primary efficacy outcomes (ie, remission and composite endpoints) and secondary outcomes (ie, mean (95\% CI) change of GFR, nephrotic syndrome, and adverse events) are presented as percentages of the risks of the event. Risk differences adjusted for effects of other factors that were imbalanced at the baseline and its $95 \%$ CI were estimated by using generalised linear models with binomial distribution and identically linked functions. For the other primary endpoints, time to remission including partial or complete remission, survival analysis and comparisons of both treatment groups using a two-sided log-rank test were performed. Competing-risk survival analyses focused on cumulative incidence of remission from treatment over time. Subhazard ratios and 95\% CI from competing-risk regressions were based on Fine and Gray's proportional subhazards model and calculated. Two-sided significance tests were used throughout and a $p$ value $<0.05$ was considered to represent a statistically significant difference.
Figure 1 Algorithm of the study showed 63 volunteers who gave written consents. Four patients did not meet the inclusion/ exclusion criteria. Fifty-nine volunteers were randomly assigned to treatment, Cyclophosphamide (CYC) or enteric-coated mycophenolic sodium (EC-MPS) using a randomisation block of four.

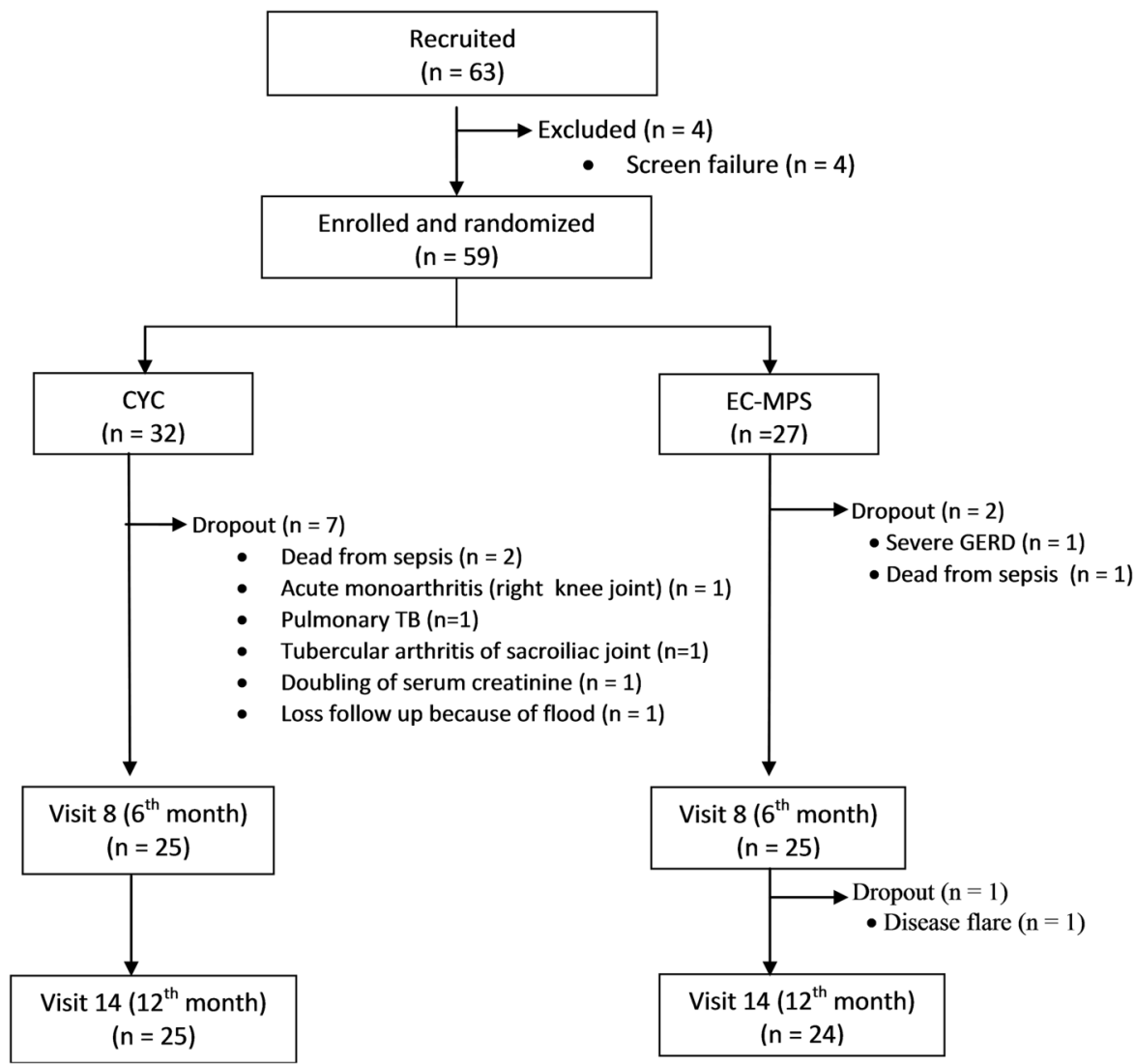


All statistical analyses were performed using the STATA V.13.0 statistical package.

\section{RESULTS}

Patients

Sixty-three volunteers from six medical centres in Thailand provided written informed consents. Four patients failed the screening due to not meeting the inclusion criteria. Fifty-nine patients were randomised, 32 in CYC group and 27 in EC-MPS group (figure 1). The patients from the EC-MPS group were numerically older, had a lower estimated GFR and had more nephrotic-range proteinuria (table 1). Eighty-three per cent were able to complete the 12-month study protocol $(78 \%$ in CYC group and $89 \%$ in EC-MPS group; figure 1).

\section{Primary outcomes}

Remission rates. At the 12th month, complete and partial remission rates were $36 \%$ and $32 \%$ in the CYC group, while those rates were $29.2 \%$ and $41.7 \%$ in the EC-MPS group (table 2). A composite of unfavourable outcomes occurred during the study of the following: nonremission, doubling of serum creatinine, intolerance to treatment or death was found in 15 of 32 patients $(46.9 \%)$ in the CYC group and 10 of 27 patients
$(37.0 \%)$ in the EC-MPS group. The per cent difference was $9.84(95 \% \quad$ CI -15.3 to $35.0 ; \quad \mathrm{p}=0.44)$. Discontinuation due to any one of the following: death, major infection, doubling of serum creatinine or loss to follow-up was found in 7 of 32 patients $(21.9 \%)$ in the CYC group and 3 of 27 patients $(11.1 \%)$ in the EC-MPS group. The per cent difference was 10.76 (95\% CI -7.8 to 29.4; $\mathrm{p}=0.26$ ). The unadjusted per cent differences of remission, discontinuation and a composite of unfavourable outcomes between both groups were not significant (table 2). Of note, the adjusted rate of discontinuation within 6 months was significantly higher in the CYC group (per cent difference 16.90, 95\% CI 1.3 to 32.4, $\mathrm{p}=0.03$; adjusted for baseline proteinuria and eGFR).

Time to achieve remission. A survival analysis of time to remission was performed with censoring for death and discontinuation and a doubling of serum creatinine. Among 59 patients, 8112 patient-days were observed. Overall remission rate was 189.11 per 100 person-years. The incidence rates of remission in the CYC and EC-MPS groups were 160.23 and 226.16 per 100 person-years. The median times to remission were not different between groups (CYC: 96 days, 95\% CI 84 to 364 and EC-MPS: 97 days, 95\% CI 37 to $189, \log$-rank test $\mathrm{p}=0.25$ ) (figure 2).

Due to the frequent occurrence of unfavourable events including serious infections or adverse events,

Table 1 Baseline characteristics of cyclophosphamide (CYC) and enteric-coated mycophenolic sodium (EC-MPS) groups

\begin{tabular}{|c|c|c|}
\hline Patients characteristic & CYC (n=32) & EC-MPS ( $n=27)$ \\
\hline Female, $\mathrm{n}(\%)$ & $32(100)$ & $26(96.3)$ \\
\hline Age at enrolment (years; mean $\pm S D$ ) & $30.2 \pm 7.0$ & $35.4 \pm 12.9$ \\
\hline Median time (min-max) of lupus nephritis (months) & $46.5(4.0-158.0)$ & $60.5(3.0-240.0)$ \\
\hline Systolic blood pressure $(\mathrm{mm} \mathrm{Hg}$; mean $\pm \mathrm{SD})$ & $132 \pm 17$ & $136 \pm 19$ \\
\hline Diastolic blood pressure (mm Hg; mean $\pm \mathrm{SD}$ ) & $85 \pm 16$ & $87 \pm 14$ \\
\hline \multicolumn{3}{|l|}{ Renal pathology, $\mathrm{n}(\%)$} \\
\hline ISN/RPS class III & $10 \%$ & $4 \%$ \\
\hline ISN/RPS class IV & $90 \%$ & $96 \%$ \\
\hline Relapse/resistant cases (\%) & $34.4 / 65.6$ & $37.0 / 63.0$ \\
\hline Serum creatinine $(\mathrm{mg} / \mathrm{dL} ;$ mean $\pm \mathrm{SD})$ & $0.90 \pm 0.42$ & $0.99 \pm 0.40$ \\
\hline eGFR by chronic kidney disease epidemiology $\left(\mathrm{mL} / \mathrm{min} / 1.73 \mathrm{~m}^{2} ;\right.$ mean $\left.\pm \mathrm{SD}\right)$ & $96.5 \pm 30.8$ & $84.7 \pm 29.9$ \\
\hline \multicolumn{3}{|l|}{ Range of eGFR $\left(\mathrm{mL} / \mathrm{min} / 1.73 \mathrm{~m}^{2} ; \mathrm{n}(\%)\right)$} \\
\hline$\geq 90 \mathrm{~mL} / \mathrm{min} / 1.73 \mathrm{~m}^{2}$ & $21(65.6)$ & $15(55.6)$ \\
\hline $60-89 \mathrm{~mL} / \mathrm{min} / 1.73 \mathrm{~m}^{2}$ & $5(15.6)$ & $4(14.8)$ \\
\hline $45-59 \mathrm{~mL} / \mathrm{min} / 1.73 \mathrm{~m}^{2}$ & $3(9.4)$ & $4(14.8)$ \\
\hline $30-44 \mathrm{~mL} / \mathrm{min} / 1.73 \mathrm{~m}^{2}$ & $2(6.3)$ & $3(11.1)$ \\
\hline $25-30 \mathrm{~mL} / \mathrm{min} / 1.73 \mathrm{~m}^{2}$ & $1(3.1)$ & $1(3.7)$ \\
\hline Haemoglobin (g/dL; mean \pm SD) & $10.8 \pm 1.9$ & $10.7 \pm 1.5$ \\
\hline Haematocrit $(\% ;$ mean \pm SD $)$ & $33.1 \pm 5.4$ & $32.6 \pm 4.5$ \\
\hline Serum albumin (g/dL; mean $\pm S D)$ & $2.88 \pm 0.72$ & $2.68 \pm 0.69$ \\
\hline \multicolumn{3}{|l|}{ Serum complement (mg/dL, mean \pm SD) } \\
\hline C3 concentration & $72.1 \pm 35.2$ & $71.8 \pm 32.6$ \\
\hline C4 concentration & $17 \pm 12.7$ & $16.4 \pm 11.4$ \\
\hline UPCR (mean $\pm S D)$ & $7.5 \pm 5.3$ & $9.0 \pm 6.1$ \\
\hline Nephrotic-range proteinuria ( $\geq 3.5$ g/day), n (\%) & $22(68.8)$ & $24(88.9)$ \\
\hline Urine red blood cell (cells/hpf; mean \pm SD) & $27.8 \pm 43.8$ & $34.4 \pm 70.2$ \\
\hline Urine white cell (cells/hpf; mean \pm SD) & $14.1 \pm 55.8$ & $10.8 \pm 29.1$ \\
\hline
\end{tabular}




\begin{tabular}{|c|c|c|c|c|c|c|c|c|c|}
\hline \multirow[b]{2}{*}{ Variables } & \multicolumn{2}{|c|}{ CYC (N=32) } & \multicolumn{2}{|c|}{ EC-MPS (N=27) } & \multicolumn{2}{|c|}{$\begin{array}{l}\text { Unadjusted percentage } \\
\text { difference between groups* }\end{array}$} & \multirow[b]{2}{*}{ p Value } & \multirow{2}{*}{$\begin{array}{l}\text { Adjusted percentage } \\
\text { difference between groups }{ }^{\star} \dagger \\
\%(95 \% \mathrm{Cl})\end{array}$} & \multirow[b]{2}{*}{ p Value } \\
\hline & No & Per cent & No & Per cent & Per cent & $(95 \% \mathrm{Cl})$ & & & \\
\hline \multicolumn{10}{|c|}{ Complete remission } \\
\hline At 6 th month & $7 / 25$ & 28.00 & $3 / 25$ & 12.00 & 16.00 & $(-5.7$ to 37.7$)$ & 0.15 & $11.2(-12.1$ to 34.6$)$ & 0.35 \\
\hline At 12th month & $9 / 25$ & 36.00 & $7 / 24$ & 29.17 & 6.83 & $(-19.3$ to 33.0$)$ & 0.61 & $-5.12(-32.7$ to 22.5$)$ & 0.72 \\
\hline \multicolumn{10}{|l|}{ Partial remission } \\
\hline At 6th month & $8 / 25$ & 32.00 & $12 / 25$ & 48.00 & -16.00 & $(-42.8$ to 10.8$)$ & 0.24 & $-12.61(-41.6$ to 16.3$)$ & 0.39 \\
\hline At 12th month & $8 / 25$ & 32.00 & $10 / 24$ & 41.67 & -9.67 & $(-34.6$ to 17.2$)$ & 0.48 & $-12.04(-40.0$ to 15.9$)$ & 0.40 \\
\hline \multicolumn{10}{|l|}{ Non-remission } \\
\hline At 6th month & $10 / 25$ & 40.00 & $10 / 25$ & 40.00 & 0.00 & $(-27.2$ to 27.2$)$ & 1.00 & $-1.20(-30.6$ to 28.3$)$ & 0.94 \\
\hline At 12th month & $8 / 25$ & 32.00 & $7 / 24$ & 29.17 & 2.83 & $(-23.0$ to 28.6$)$ & 0.83 & $11.40(-14.4$ to 37.1$)$ & 0.39 \\
\hline \multicolumn{10}{|l|}{ Discontinuation $\ddagger$} \\
\hline 0-6th month & $7 / 32$ & 21.88 & $2 / 27$ & 7.41 & 14.47 & $(-2.9$ to 31.9$)$ & 0.10 & 16.90 (1.3 to 32.4$)$ & 0.03 \\
\hline $7-12$ th month & $0 / 25$ & 0.00 & $1 / 25$ & 4.00 & -4.00 & $(-11.7$ to 3.7$)$ & 0.31 & $-0.40(-11.5$ to 3.5$)$ & 0.30 \\
\hline 0-12th month & $7 / 32$ & 21.88 & $3 / 27$ & 11.1 & 10.76 & $(-7.8$ to 29.4$)$ & 0.26 & $14.11(-3.1$ to 31.3$)$ & 0.11 \\
\hline \multicolumn{10}{|c|}{ Composite outcome§ } \\
\hline 0-6th month & $17 / 32$ & 53.13 & $12 / 27$ & 44.44 & 8.68 & $(-16.8$ to 34.2$)$ & 0.51 & $10.68(-15.7$ to 37.1$)$ & 0.43 \\
\hline $7-12$ th month & $8 / 25$ & 32.00 & $8 / 25$ & 32.00 & 0.00 & $(-25.9$ to 25.9$)$ & 1.00 & $6.31(-20.4$ to 33.0$)$ & 0.64 \\
\hline 0-12th month & $15 / 32$ & 46.88 & $10 / 27$ & 37.04 & 9.84 & $(-15.3$ to 35.0$)$ & 0.44 & $12.8(-11.8$ to 37.4$)$ & 0.31 \\
\hline
\end{tabular}


Figure 2 Time to first remission during 12 months of the study according to treatment groups: group 1; CYC, cyclophosphamide and group 2; EC-MPS, enteric-coated mycophenolate sodium.

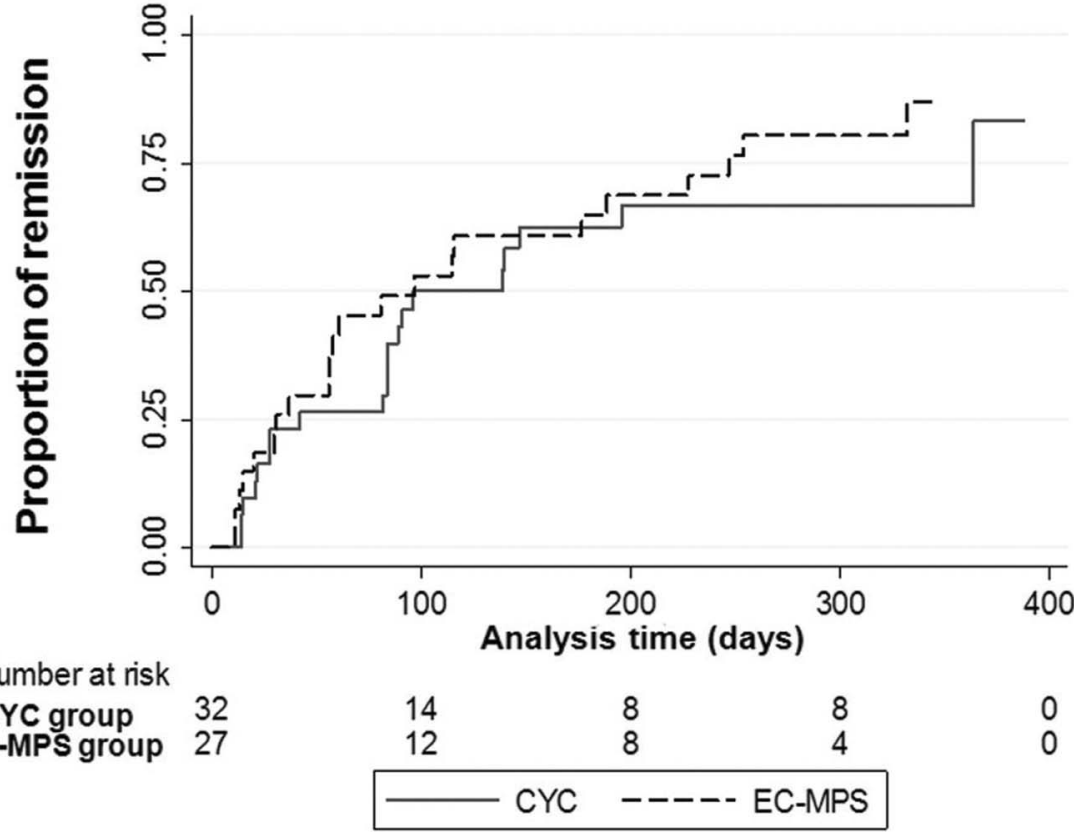

doubling of serum creatinine, and death, an analysis by competing-risk analysis was performed. The competing-risk regression model revealed an unadjusted subhazard ratio for the remission rates between EC-MPS and CYC that was 1.60 (95\% CI 0.88 to 2.90), $\mathrm{p}=0.12$ and the subhazard ratio adjusted for baseline proteinuria and eGFR was 1.95 (95\% CI 1.01 to 3.78), $\mathrm{p}=0.048$. The cumulative incidence of remission of the treatment groups is shown in figure 3 .

\section{Secondary outcomes}

The secondary outcomes, that is, percentage of nephrotic syndrome, renal impairment (change of GFR as compared with baseline) and serious adverse events are shown in table 3 . The percent differences of nephrotic syndrome and serious adverse events between both groups were not of statistical significance. The changes of GFR at the 6th and 12th months from baseline were not significantly different. The percentage of serious fatal and non-fatal infections was, however, higher in the CYC group $(15.63 \%$ vs $3.7 \%$, the per cent difference adjusted for baseline proteinuria 13.35 (95\% CI 0.3 to 26.4), $\mathrm{p}=0.04$ ). Other adverse events included one case of diarrhoea, two of gastro-oesophageal reflux in EC-MPS group, two cases of leucopenia in the CYC group and two episodes of renal flare in each group.

\section{DISCUSSION}

This study was aimed to demonstrate the superiority of EC-MPS for the treatment of relapsed or the resistant type of proliferative LN. It is the first randomised
Figure 3 Cumulative incidence of remission according to treatment groups taking competing risks into account. CYC, cyclophosphamide; EC-MPS, enteric-coated mycophenolate sodium.

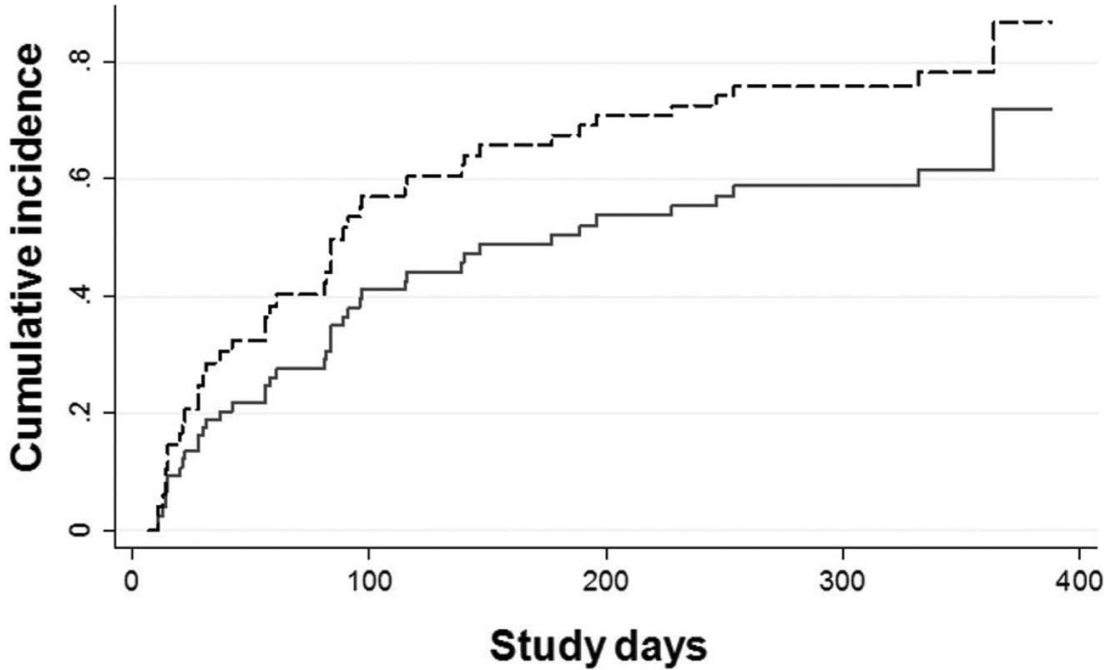

CYC ----EC-MPS 


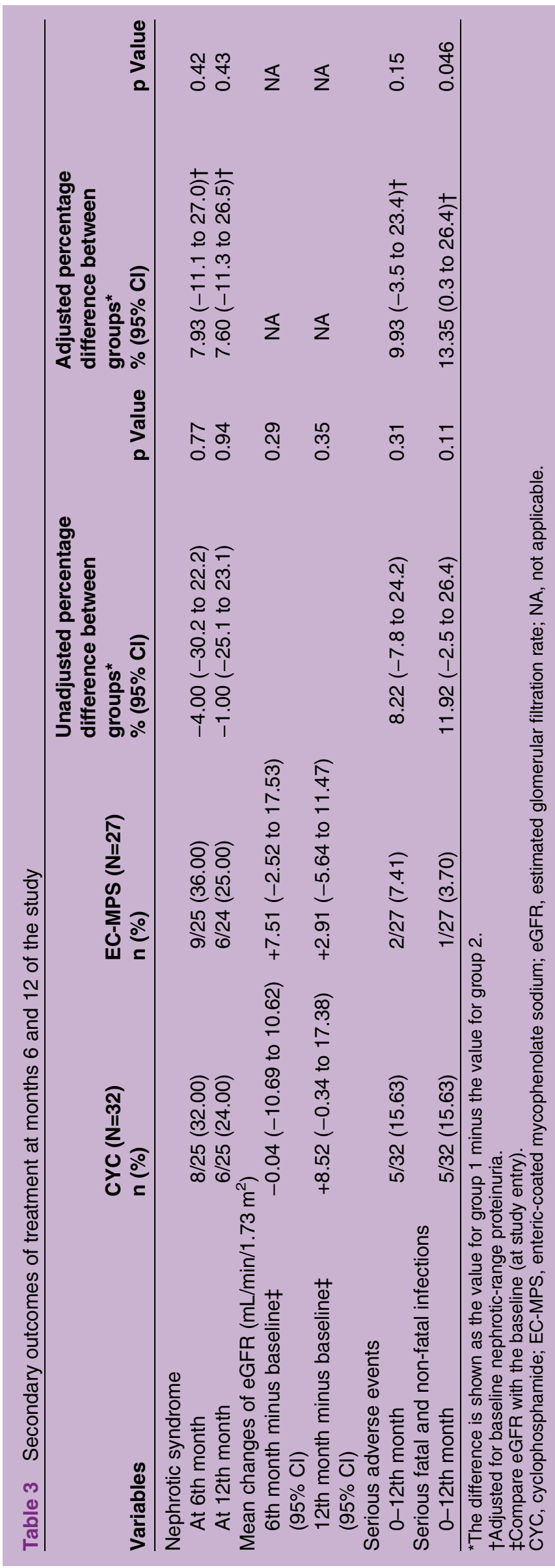

controlled trial to evaluate the efficacy of EC-MPS on this difficult-to-treat type of $\mathrm{LN}$ and it is similar to a reallife clinical setting. The study was prematurely terminated by the DSMB due to a high incidence $(>10 \%)$ of serious adverse events from any treatment arm. Owing to the early stopping among 59 participants, EC-MPS provided $71 \%$ while CYC had $68 \%$ of combined partial and complete responses. Although the study was unable to reach the primary endpoint, there was a higher percentage of unfavourable end points from the CYC treatment arm $(46.9 \%$ vs $37 \%)$. Furthermore, the serious adverse events including serious infections or death were numerically high in the CYC arm and were the reason of early termination of the study. This reflects intolerance to the CYC during the first 6 months of the induction period.

The characteristic feature of $\mathrm{LN}$ in Asians is aggressive disease and has a high rate of renal relapse as shown in many reports. ${ }^{4-7}$ The number of relapses have been associated with the increased risk of end-stage renal disease and death. ${ }^{3}{ }^{12-15}$ The multinational, multicentre study between MMF versus CYC treatment (ALMS trial) has shown an equal efficacy of both induction treatments across ethnic groups ${ }^{19}$ while MMF was superior particularly in African and Hispanic ethnic populations. $^{21}{ }^{22}$ For Asian ethnic populations, a study on newly diagnosed LN found comparable efficacy between MMF (2 g/day) and CYC for induction treatment. ${ }^{23}$ In this study, the participants were considered in the category of the most severe and grave prognosis group of LN. Given the enrolment criteria that included biopsyproven class III/IV LN within 16 weeks of randomisation, all participants were confirmed in the most active stage. All had experienced CYC therapy or received three consecutive doses of IVCY. Furthermore, some patients were recovered from rapidly progressive glomerulonephritis (RPGN) and their estimated GFR had returned to more than $25 \mathrm{~mL} / \mathrm{min} / 1.73 \mathrm{~m}^{2}$ within 12 weeks. It should be emphasised that RPGN is considered an exclusion criteria in most randomised controlled studies because it is the most difficult-to-treat condition. Moreover, a number of patients received 1.5$2 \mathrm{~g}$ of intravenous methylprednisolone due to the crescentic formations seen in renal histopathology. This difficult-to-treat type of LN could be the reason for the high incidence of serious adverse events that eventually led to early termination of this study.

Although only 59 patients were able to finish the 12 months of the study, the efficacy and safety profiles of both regimens should be reported with cautious interpretation. EC-MPS treatment provided a comparable rate of overall response (difference between groups was $2.83 \%, 95 \%$ CI -23 to 28.6 ). This has confirmed previous observations of the efficacy of EC-MPS. ${ }^{30}$ The overall response rate of EC-MPS treatment increased from $60 \%$ to $71 \%$ at the 6th and 12th month of study. The overall response rate was also increased in the IVCY followed by EC-MPS treatment from $60 \%$ to $68 \%$ at 6 and 
12 months. Therefore, the continuation of or the switching to mycophenolate regimen at the 24th week increased the response rate, which has been reported in many studies. ${ }^{18-21} 36$ Furthermore, a switching to EC-MPS after the 6th month may not harm the kidney function, despite a short observation period. In the first 24-weeks of the study, a number of discontinuations from the study due to intolerance to treatment or serious adverse events were also observed by these current investigators (11\% vs $19 \%$ in EC-MPS and CYC arms). There were numerically higher rates of serious infection (sepsis) and death ( $\mathrm{n}=2)$ in the CYC arm (one of relapse and one of resistant type). After adjusting the baseline, these adverse events were significantly higher in the CYC arm. There were serious infections $(n=3)$ and tuberculosis $(\mathrm{n}=2)$ in patients receiving $\mathrm{CYC}$, and four of those five cases were from resistant group. EC-MPS treatment was better tolerated in this study although one fatal case was from resistant case. Concerning infectious risks of CYC in those with three consecutive doses, CYC was withheld during the screening period for at least 6 weeks. All patients with active infection were a screening failure. Nonetheless, the adverse events could be due to concomitant use of steroids.

It should be noted that the main limitation of this study could be the early termination. The number of participants had not reached the planned target. Nevertheless, this was due to a high rate of death and infection in the CYC arm. This could reflect the high risk of continuous use of intravenously CYC in relapsed or resistant LN. This risk should be written in the guidelines or recommendations since Asian patients often exposed to high cumulative dose of CYC. In the competing-risk analysis, there was a significantly higher cumulative rate and adjusted subhazard ratio of remission in the EC-MPS group (figure 3). Another limitation of this study was found in the secondary outcomes (table 3). The estimated GFR was significantly lower in EC-MPS group partly due to the imbalance of the randomisation. However, both treatments did not differently affect GFR.

Due to an early termination of the study, the target sample size could not be reached. The conclusion could be drawn cautiously with this limitation. EC-MPS plus steroids could be an alternative treatment of choice for difficult-to-treat LN. EC-MPS at the dosage of $1440 \mathrm{mg}$ per day for 6 months was well tolerated in Asians and provided acceptable efficacy compared with intravenous CYC.

\footnotetext{
Author affiliations

${ }^{1}$ Department of Medicine, Khon Kaen University, Khon Kaen, Thailand ${ }^{2}$ Department of Medicine, Khon Kaen Regional Hospital, Khon Kaen, Thailand ${ }^{3}$ Department of Medicine, Nopparat Rajathani Hospital, Bangkok, Thailand ${ }^{4}$ Department of Medicine, Phramongkutklao Hospital, Bangkok, Thailand ${ }^{5}$ Department of Medicine, Thammasart University, Pathumthani, Thailand ${ }^{6}$ Medical University Research Network of the Consortium of Thai Medical Schools (MedResNet), Thailand
}

${ }^{7}$ Chulalongkorn Clinical Research Center (ChulaCRC), Chulalongkorn University, Bangkok, Thailand

${ }^{8}$ Department of Biostatistics and Demography, Faculty of Public Health, Khon Kaen University, Khon Kaen, Thailand

${ }^{9}$ Department of Medicine, Faculty of Medicine, Chulalongkorn University, Bangkok, Thailand

${ }^{10}$ Center of Excellence in Immunology and Immune-mediated Diseases, Chulalongkorn University, Bangkok, Thailand

Acknowledgements The authors would like to thank Professor Pyatat Tatsanavivat and the members of Clinical Research Collaboration Network (CRCN); currently Medical Research Network (MedResNet), Ms Sasiwimol Ubolyam of the Chula Clinical Research Laboratory for their support. We would like to acknowledge Professor James A Will for editing the manuscript via the publication clinic KKU, Thailand.

Contributors Protocol and manuscript writing (SA, BT, YA), data management and trial monitoring (WP, CR), statistical analysis (SA, TP, BT), site investigators (JW, SC, BS, OT, TP, SA, YA), contract-research organisation: MedResNet Medical Research Foundation (MRF), Thailand.

Funding Grant supported from Health Intervention and Technology Assessment Program (HITAP), Matching Fund (Faculty of Medicine, Chulalongkorn University) and Investigator-initiated trial (CERL080ATH04T).

Competing interests YA (Speaker/Advisory of Astellas, Novartis, Pfizer).

Patient consent Obtained.

Ethics approval Ethical Committee of Local Institutes (IRB No. 313/52).

Provenance and peer review Not commissioned; externally peer reviewed.

Data sharing statement No additional data are available.

Open Access This is an Open Access article distributed in accordance with the Creative Commons Attribution Non Commercial (CC BY-NC 4.0) license, which permits others to distribute, remix, adapt, build upon this work noncommercially, and license their derivative works on different terms, provided the original work is properly cited and the use is non-commercial. See: http:// creativecommons.org/licenses/by-nc/4.0/

\section{REFERENCES}

1. Ortega LM, Schultz DR, Lenz O, et al. Lupus nephritis: pathologic features, epidemiology and a guide to therapeutic decisions. Lupus 2010;19:557-74.

2. Gamba G, Quintanilla L, del Bosque MD, et al. Clinical course and prognostic factors in lupus nephropathy. Rev Invest Clin 2000;52:397-405

3. Sprangers B, Monahan M, Appel GB. Diagnosis and treatment of lupus nephritis flares--an update. Nat Rev Nephrol 2012;8:709-17.

4. Mok CC, Yap DY, Navarra SV, et al. Overview of lupus nephritis management guidelines and perspective from Asia. Nephrology (Carlton) 2014;19:11-20.

5. Jakes RW, Bae S-C, Louthrenoo W, et al. Systematic review of the epidemiology of systemic lupus erythematosus in the Asia-Pacific region: prevalence, incidence, clinical features, and mortality. Arthritis Care Res 2012;64:159-68.

6. Saxena R, Mahajan T, Mohan C. Lupus nephritis: current update. Arthritis Res Ther 2011;13:240.

7. Yap DY, Chan TM. Treatment of lupus nephritis: practical issues in Asian countries [published online ahead of print June 26, 2014]. Int $J$ Rheum Dis 2015;18:138-45.

8. Hiramatsu N, Kuroiwa $\mathrm{T}$, Ikeuchi $\mathrm{H}$, et al. Revised classification of lupus nephritis is valuable in predicting renal outcome with an indication of the proportion of glomeruli affected by chronic lesions. Rheumatology (Oxford) 2008:47:702-7.

9. Markowitz GS, D'Agati VD. Classification of lupus nephritis. Curr Opin Nephrol Hypertens 2009;18:220-5.

10. Conlon PJ, Fischer CA, Levesque MC et al. Clinical, biochemical and pathological predictors of poor response to intravenous cyclophosphamide in patients with proliferative lupus nephritis. Clin Nephrol 1996;46:170-5.

11. Shayakul C, Ong-aj-yooth L, Chirawong $P$, et al. Lupus nephritis in Thailand: clinicopathologic findings and outcome in 569 patients. Am J Kidney Dis 1995;26:300-7. 
12. Kalloo S, Aggarwal N, Mohan P, et al. Lupus nephritis: treatment of resistant disease. Clin J Am Soc Nephrol 2013;8:154-61.

13. Illei GG, Takada K, Parkin D, et al. Renal flares are common in patients with severe proliferative lupus nephritis treated with pulse immunosuppressive therapy: long-term followup of a cohort of 145 patients participating in randomized controlled studies. Arthritis Rheum 2002;46:995-1002.

14. Moroni G, Quaglini S, Maccario M, et al. "Nephritic flares" are predictors of bad long-term renal outcome in lupus nephritis. Kidney Int 1996;50:2047-53.

15. Sidiropoulos PI, Kritikos HD, Boumpas DT. Lupus nephritis flares. Lupus 2005;14:49-52.

16. Henderson LK, Masson P, Craig JC, et al. Induction and maintenance treatment of proliferative lupus nephritis: a meta-analysis of randomized controlled trials. Am J Kidney Dis 2013;61:74-87.

17. Mak A, Cheak AA, Tan JY, et al. Mycophenolate mofetil is as efficacious as, but safer than, cyclophosphamide in the treatment of proliferative lupus nephritis: a meta-analysis and meta-regression. Rheumatology (Oxford) 2009;48:944-52.

18. Yap DY, Ma MK, Mok MM, et al. Long-term data on corticosteroids and mycophenolate mofetil treatment in lupus nephritis. Rheumatology (Oxford) 2013;52:480-6.

19. Appel GB, Contreras G, Dooley MA, et al. Mycophenolate mofetil versus cyclophosphamide for induction treatment of lupus nephritis. J Am Soc Nephrol 2009;20:1103-12.

20. Houssiau FA, D'Cruz D, Sangle S, et al. Azathioprine versus mycophenolate mofetil for long-term immunosuppression in lupus nephritis: results from the MAINTAIN Nephritis Trial. Ann Rheum Dis 2010;69:2083-9.

21. Dooley MA, Jayne D, Ginzler EM, et al. Mycophenolate versus azathioprine as maintenance therapy for lupus nephritis. $N$ Engl $J$ Med 2011;365:1886-95.

22. Ginzler EM, Dooley MA, Aranow C, et al. Mycophenolate mofetil or intravenous cyclophosphamide for lupus nephritis. $N$ Engl $J$ Med 2005;353:2219-28.

23. Ong LM, Hooi LS, Lim TO, et al. Randomized controlled trial of pulse intravenous cyclophosphamide versus mycophenolate mofetil in the induction therapy of proliferative lupus nephritis. Nephrology (Carlton) 2005;10:504-10.
24. Contreras G, Tozman E, Nahar N, et al. Maintenance therapies for proliferative lupus nephritis: mycophenolate mofetil, azathioprine and intravenous cyclophosphamide. Lupus 2005;14(suppl 1):s33-8.

25. El-Shafey EM, Abdou SH, Shareef MM. Is mofetil superior to pulse intravenous cyclophosphamide for induction therapy of proliferative lupus nephritis in Egyptian patients? Clin Exp Nephrol 2010;14:214-21.

26. Contreras G, Pardo V, Leclercq B, et al. Sequential therapies for proliferative lupus nephritis. N Engl J Med 2004;350:971-80.

27. Mohara A, Pérez Velasco R, Praditsitthikorn N, et al. A cost-utility analysis of alternative drug regimens for newly diagnosed severe lupus nephritis patients in Thailand. Rheumatology (Oxford) 2014:53:138-44.

28. Dooley MA, Cosio FG, Nachman PH, et al. Mycophenolate mofetil therapy in lupus nephritis: clinical observations. J Am Soc Nephrol 1999;10:833-9.

29. Kingdon EJ, McLean AG, Psimenou E, et al. The safety and efficacy of MMF in lupus nephritis: a pilot study. Lupus 2001;10:606-11.

30. Traitanon $\mathrm{O}$, Avihingsanon $\mathrm{Y}$, Kittikovit $\mathrm{V}$, et al. Efficacy of enteric-coated mycophenolate sodium in patients with resistant-type lupus nephritis: a prospective study. Lupus 2008;17:744-51.

31. Rivera F, Mérida E, Illescas ML, et al. Mycophenolate in refractory and relapsing lupus nephritis. Am J Nephrol 2014;40:105-12.

32. Karim MY, Alba P, Cuadrado MJ, et al. Mycophenolate mofetil for systemic lupus erythematosus refractory to other immunosuppressive agents. Rheumatology (Oxford) 2002:41:876-82.

33. Kapitsinou PP, Boletis JN, Skopouli FN, et al. Lupus nephritis: Treatment with mycophenolate mofetil. Rheumatology (Oxford) 2004;43:377-80.

34. Lai KN, Tang SC, Mok CC. Treatment for lupus nephritis: a revisit. Nephrology (Carlton) 2005;10:180-8.

35. Austin HA III, Klippel JH, Balow JE, et al. Therapy of lupus nephritis. Controlled trial of prednisone and cytotoxic drugs. N Engl J Med 1986;314:614-19.

36. Kittanamongkolchai W, Rukrung C, Supasiri T, et al. Therapeutic drug monitoring of mycophenolate mofetil for the treatment of severely active lupus nephritis. Lupus 2013;22:727-32. 